\title{
Silicon-on-insulator ring-shaped photonic crystal waveguides for refractive index sensing
}

Pu, Minhao; Liu, Liu; Frandsen, Lars Hagedorn; Ou, Haiyan; Yvind, Kresten; Hvam, Jørn Märcher

Published in:

2010 Conference on (OFC/NFOEC) Optical Fiber Communication (OFC), collocated National Fiber Optic Engineers Conference

Link to article, DOI:

10.1364/nfoec.2010.jwa20

Publication date:

2010

Document Version

Publisher's PDF, also known as Version of record

Link back to DTU Orbit

Citation (APA):

Pu, M., Liu, L., Frandsen, L. H., Ou, H., Yvind, K., \& Hvam, J. M. (2010). Silicon-on-insulator ring-shaped photonic crystal waveguides for refractive index sensing. In 2010 Conference on (OFC/NFOEC) Optical Fiber Communication (OFC), collocated National Fiber Optic Engineers Conference (pp. 1-3). [5465667] IEEE. https://doi.org/10.1364/nfoec.2010.jwa20

\section{General rights}

Copyright and moral rights for the publications made accessible in the public portal are retained by the authors and/or other copyright owners and it is a condition of accessing publications that users recognise and abide by the legal requirements associated with these rights.

- Users may download and print one copy of any publication from the public portal for the purpose of private study or research.

- You may not further distribute the material or use it for any profit-making activity or commercial gain

- You may freely distribute the URL identifying the publication in the public portal 


\title{
Silicon-on-Insulator Ring-Shaped Photonic Crystal Waveguides for Refractive Index Sensing
}

\author{
Minhao Pu ${ }^{1}$, Liu Liu ${ }^{1}$, Lars H. Frandsen ${ }^{2}$, Haiyan $\mathrm{Ou}^{1}$, Kresten Yvind ${ }^{1}$ and Jørn M. Hvam ${ }^{1}$ \\ ${ }^{1}$ DTU Fotonik, Department of Photonics Engineering, Technical University of Denmark, DK-2800 Lyngby, Denmark \\ ${ }^{2}$ NKT Photonics, Blokken 84, DK-3460 Birkeroed, Denmark \\ Email:mipu@fotonik.dtu.dk
}

\begin{abstract}
We present an integrated refractive index sensor based on a ring-shaped photonic crystal waveguide. We measured the shift in cut-off wavelength for different ambient refractive indices and we observed a sensitivity of $\Delta \lambda / \Delta n=110 \mathrm{~nm} / \mathrm{RIU}$.

(C)2010 Optical Society of America

OCIS codes: (130.3120) Integrated optics devices; (130.6010) Sensors; (230.5298) Photonic crystals.
\end{abstract}

\section{Introduction}

The application of a refractive index (RI) sensor has gained a high degree of interest within the last decade since it can be applied to measure very small changes in temperature, pressure, humidity, chemical composition, etc. Many integrated optical sensors based on Mach-Zehnder interferometers [1], directional couplers [2], micro-rings [3], and disk resonators [4] can be made compact and integrated with both optical and electric components onto one single chip and have already been demonstrated. Recently, ultra-compact and highly sensitive sensors based on planar photonic crystal waveguides (PhCW) [5] and cavities [6] fabricated on silicon-on-insulator (SOI) wafers have also been demonstrated for e.g. bio-sensing. Here, we present an integrated RI sensor based on a ring-shaped photonic crystal waveguide (RPhCW) [7] which provide higher sensitivity than RI sensors based on PhCW with circular holes [5]. We experimentally demonstrated a sensitivity of $\Delta \lambda / \Delta n=110 \mathrm{~nm} / \mathrm{RIU}$ for the device.

\section{Design and simulation}

The proposed RPhCW sensor consists of a hexagonal lattice (pitch $\Lambda$ ) of ring-shaped holes which are defined by their outer $\left(R_{o}\right)$ and inner $\left(R_{i}\right)$ radii. As illustrated in Fig. 1(left), the RPhCW is patterned in a 340-nm thick top silicon layer of a SOI chip, and a W1 RPhCW was formed by omitting one row of the holes. This line defect introduces waveguide modes within the photonic band gap (PBG) as shown in Fig. 1(right). The band diagram is also presented for the $\mathrm{RPhCW}$ with $R_{o}=0.384 \Lambda$ and $R_{i}=0.21 \Lambda$ for two different ambient RI with $\mathrm{n}=1$ (blue) and $\mathrm{n}=1.1$ (red). It is seen that as the ambient RI increases, the even waveguide mode denoted as a solid continuous line is pulled down to lower frequencies which makes the cut-off wavelength red-shift in the transmission spectra. This property of the $\mathrm{RPhCW}$ is used as the sensor mechanism.
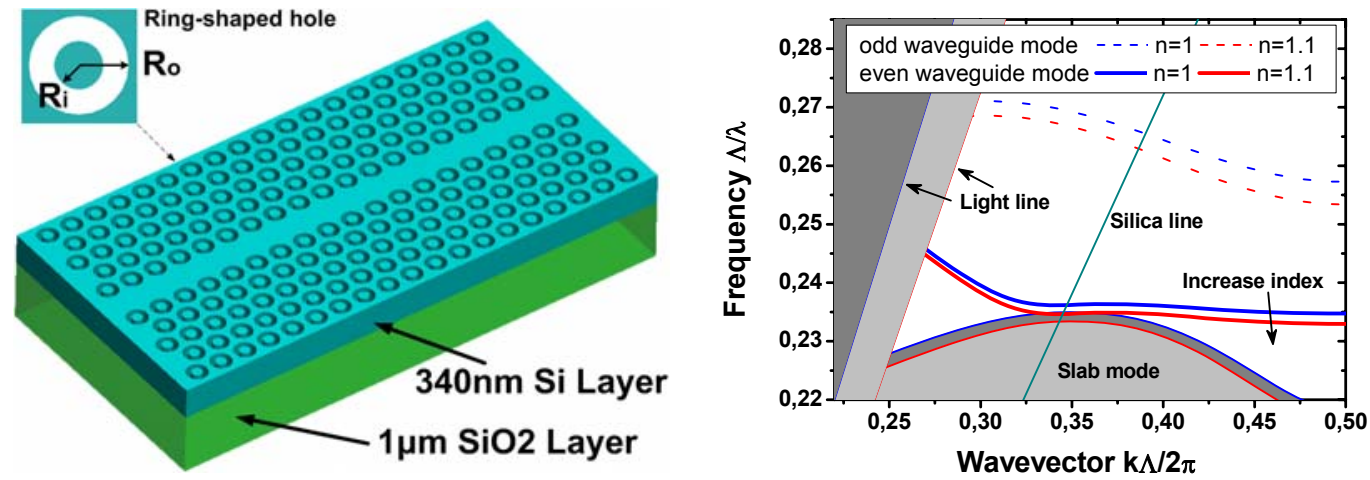

Fig. 1. Schematic diagram of the RPhCW (left), and Band diagram for RPhCW (right) with $R_{o}=0.3844$ and $R_{i}=0.214$.

Figure 2(a) shows the quasi transverse-electric (TE) mode transmission spectra calculated by three-dimensional (3D) finite-difference time-domain (FDTD) modelling. Spectra were calculated for four ambient RIs, i.e., air ( $n=1$ ), water $(n=1.315)$, ethanol $(n=1.344)$ and isopropanol (IPA) $(n=1.363)$ [8]. It is seen that the cut-off wavelength shifts to longer wavelength with increasing ambient RI. The 3D simulation was performed using the MIT Photonic Bands software both for the $\mathrm{RPhCW}$ and a conventional $\mathrm{PhCW}$ to make a comparison on sensitivity. The geometrical parameters of $R_{i}=0.324 \Lambda$ for the conventional $\mathrm{PhCW}$ was used to ensure the same air filling factor as the $\mathrm{RPhCW}$. 
Figure 2(b) shows the cut-off wavelength shift against ambient RI change for two different waveguides. From the simulation, we find that the sensitivities of the $\mathrm{PhCW}$ and the $\mathrm{RPhCW}$ are $63 \mathrm{~nm} / \mathrm{RIU}$ and $128 \mathrm{~nm} / \mathrm{RIU}$, respectively. The sensitivity of the $\mathrm{RPhCW}$ is twice as large as that of the conventional $\mathrm{PhCW}$. Since the sensitivity is determined by the overlap between the optical field of the waveguide and the ambient, we also compared the mode field distribution of the two waveguide structures at the same wavevector of $0.3(k \Lambda / 2 \pi)$. From the Fig 2 (c), one can find that the mode in the PhCW mainly penetrates only into the two rows of holes adjacent to the waveguide core while the mode in the RPhCW penetrates highly into the cladding of the waveguide. Besides, the intensity of the mode field tends to be more localized in the ring-shaped holes of the $\mathrm{RPhCW}$. Therefore, a larger overlap between the optical field and the ambient in $\mathrm{RPhCW}$ can be expected, which results in a higher sensitivity. Furthermore, we have also investigated the RPhCWs with different ring-gap widths $\left(R_{o}-R_{i}\right)$ under water infiltration, as shown in the inset of Fig. 2(b). It is found that the sensitivity can be further increased by using narrower ring-gap widths.

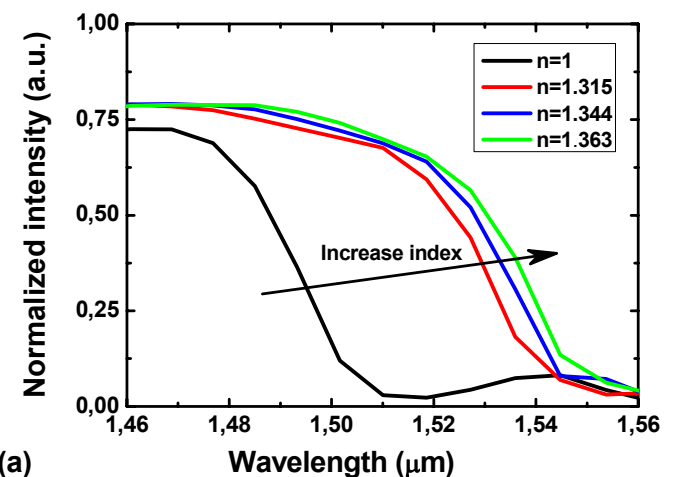

(a)

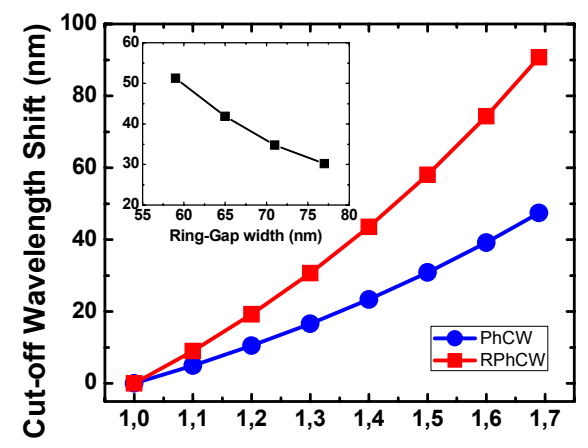

(b)

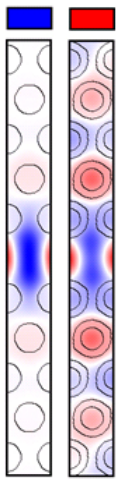

(c)

Fig. 2. (a) Simulated transmission for the RPhCW $\left(R_{o}=165 \mathrm{~nm}, R_{i}=76 \mathrm{~nm}\right.$ and $\left.\Lambda=405 \mathrm{~nm}\right)$, (b) Simulated cut-off wavelength shift with different ambient RI and different ring-gap width with infiltration of water (inset), (c) The mode field distribution for RPhCW (right) and PhCW (left).

\section{Fabrication}

The RPhCW sensor was fabricated in SOI material having a top silicon thickness of $340 \mathrm{~nm}$ and $1 \mu \mathrm{m}$ of buried silicon dioxide. Diluted (1:1 in anisole) electron-beam resist ZEP520A was spun onto the wafer to create a $\sim 110 \mathrm{~nm}$ thick masking layer. RPhCWs were defined in the ZEP520A layer by utilizing electron-beam lithography (JEOL JBX-9300FS). The patterns were subsequently transferred to the top silicon layer by employing inductively coupled plasma reactive ion etching (ICP-RIE). We fabricated RPhCWs with a fixed lattice constant $(\Lambda=405 \mathrm{~nm})$ and different ring-gap widths. Figure 3(a) shows the scanning electron micrograph (SEM) image for a RPhCW. A $20 \mu m$ long RPhCW was butt-coupled to $450 \mathrm{~nm}$ wide access ridge waveguides at both edges, one of which is shown in Fig. 3(b). The ridge waveguides were tapered to the edges of the sample for better mode matching with the lensed fibers used in the characterization. A detailed image for the ring-shaped holes with the smallest gap that can be fabricated without shape degradation is shown in Fig. 3(c).
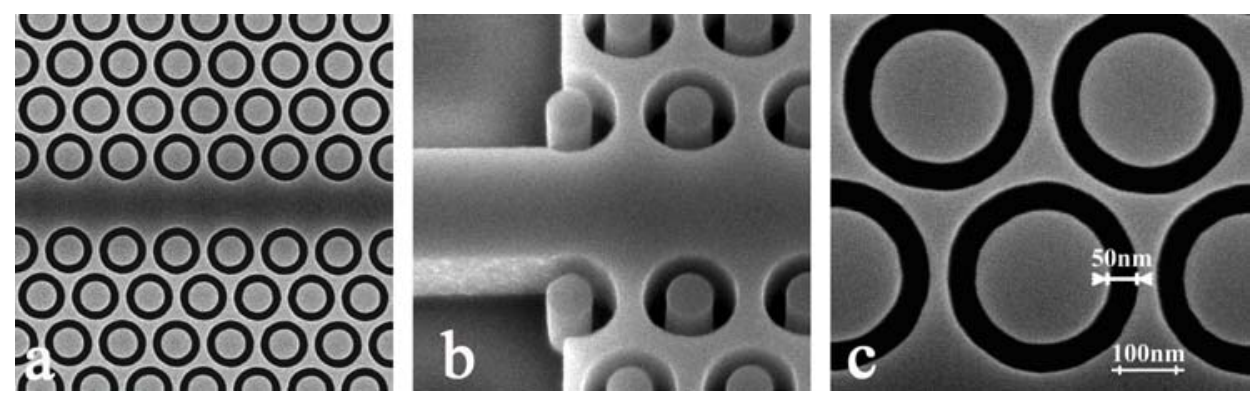

Fig. 3. Scanning electron micrograph pictures of (a) the RPhCW $\left(R_{o}=160 \mathrm{~nm}, R_{i}=90 \mathrm{~nm}\right.$ and $\left.\Lambda=405 \mathrm{~nm}\right)$, (b) the connection region between ridge waveguide and the $\mathrm{RPhCW}$ and (c) the 50 -nm width ring-shaped holes.

\section{Measurement}

Light transmission experiment was performed, where light from a tunable laser source (1520 - 1620nm) was launched into the sample and collected by an optical spectral analyzer to record the transmission spectrum. The polarization of the input light was adjusted to the quasi-TE mode with a fiber polarization controller. 
We tested the waveguide sensor with infiltrations of air, de-ionized (DI) water, ethanol and IPA. Figure 4(a) shows the recorded transmission spectra for a fabricated $\mathrm{RPhCW}$ with $R o=160 \mathrm{~nm}$ and $R i=90 \mathrm{~nm}$. The measured curves in air, DI water, ethanol and IPA are denoted with the black, red, blue and green solid lines, respectively. The cut-off wavelength was read out at the transmission of $-35 d B$ as shown with the dashed line in Fig. 4(a). The cut-off wavelength of the RPhCW shifted by $25.2 \mathrm{~nm}, 28.3 \mathrm{~nm}$ and $33.8 \mathrm{~nm}$ relative to that with air infiltration. As a comparison, we repeated the measurement for a conventional PhCW with the same air filling factor $(R=123 \mathrm{~nm}$, $\Lambda=380 \mathrm{~nm}$ ). With infiltration of the same liquids, the cut-off wavelength of the PhCW shifted by $17.8 \mathrm{~nm}$ (water), $20.3 \mathrm{~nm}$ (ethanol) and $21.9 \mathrm{~nm}$ (IPA). Thus, the RPhCW offers higher sensitivity.

Figure 4(b) shows the change of the cut-off wavelength with different ambient RIs. It is seen that the measured and simulated results are consistent for the $\mathrm{PhCW}$, but there exists a small discrepancy for the RPhCW. This is probably because the RPhCW was only partially infiltrated by those liquids due to the narrow gaps of the rings and the liquid surface tension. Furthermore, the sensitivity of the RPhCW with different ring-gap widths with IPA infiltration was measured, as shown in the inset of Fig. 4(b). We observed the largest cut-off wavelength shift of $40 \mathrm{~nm}$ for the RPhCW with $90 \mathrm{~nm}$ ring gap. This corresponds to a sensitivity of $\Delta \lambda / \Delta n=110 \mathrm{~nm} / \mathrm{RIU}$. However, contrary to the numerical prediction, the sensitivity does not increase when decreasing the ring-gap width. One possible reason is that the infiltration becomes more difficult for a narrower gap. Additionally, the silicon material in the narrower ring-shaped holes $(<90 \mathrm{~nm})$ may only have been partially etched due to the RIE lag effect. The incomplete etching for the $\mathrm{RPhCW}$ is detrimental as predicted by our simulation. This also makes infiltration of liquid more difficult which will result in a reduced sensitivity. To further improve the sensitivity, slight over etching for RPhCWs with narrow ring-gap width is necessary, and a flow-injection system [9] could be used to facilitate the liquid infiltration.
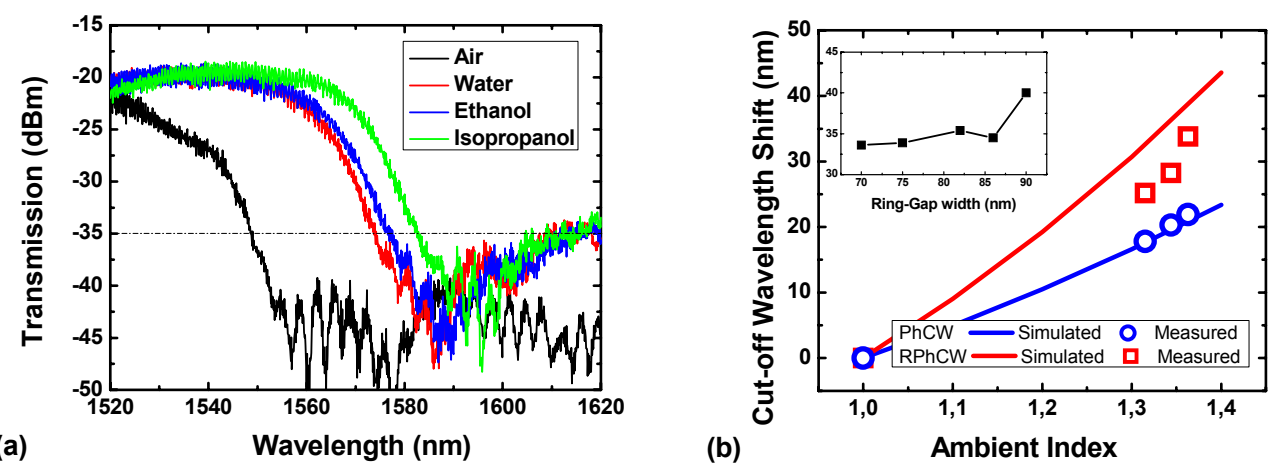

Fig. 4. (a) Measured transmission spectra of the RPhCW $\left(R_{o}=160 \mathrm{~nm}, R_{i}=90 \mathrm{~nm}\right.$ and $\left.\Lambda=405 \mathrm{~nm}\right)$ with different infiltrations. (b) Measured cut-off wavelength shift with different ambient RI (open circles and open squares) and different ring-gap width with infiltration of IPA (inset).

\section{Conclusion}

We have demonstrated an RPhCW-based RI sensor in SOI material. We have reported a sensitivity of $110 \mathrm{~nm} / \mathrm{RIU}$ which is nearly twice as large as that of the conventional PhCW-based RI sensor. We believe the RPhC structure is very promising for future sensing applications.

\section{Reference}

[1] R. G. Heideman, R. P. H. Kooyman, and J. Greve, "Performance of a highly sensitive optical waveguide Mach-Zehnder interferometer immunosensor", Sens. Actuators B 10, 209 (1993).

[2] B. J. Luff, R. D. Harris, and J. S. Wilkinson, "Integrated-optical directional coupler biosensor”, Opt. Lett. 21, 618 (1996).

[3] A. Schweinsberg, S. Hocdé, N. N. Lepeshkin, R. W. Boyd, C. Chase, and J. E. Fajardo, "An environmental sensor based on an integrated optical whispering gallery mode disk resonator", Sens. Actuators B 123, 727 (2007).

[4] C.A. Barrios, K.B. Gylfason, B. S nchez, A. Griol, H. Sohlstr m, M. Holgado, and R. Casquel, "Slot-waveguide biochemical sensor," Optics Letters, vol. 32, Nov. 2007, pp. 3080-3082.

[5] D.F. Dorfner, T. Hurlimann, T. Zabel, L.H. Frandsen, G. Abstreiter, and J.J. Finley, "Silicon photonic crystal nanostructures for refractive index sensing," Applied Physics Letters, vol. 93, Nov. 2008, pp. 181103-3..

[6] N. Skivesen, A. T tu, M. Kristensen, J. Kjems, L.H. Frandsen, and P.I. Borel, “Photonic-crystal waveguide biosensor," Optics Express, vol. 15, Mar. 2007, pp. 3169-3176.

[7] A. Säynätjoki, M. Mulot, K. Vynck, D. Cassagne, J. Ahopelto, and H. Lipsanen, "Properties, applications and fabrication of photonic crystals with ring-shaped holes in silicon-on-insulator," Photonics and Nanostructures - Fundamentals and Applications, vol. 6, Apr. 2008 , pp. $42-46$.

[8] C. Kim and C.B. Su, "Measurement of the refractive index of liquids at 1.3 and 1.5 micron using a fibre optic Fresnel ratio meter", Meas. Sci. Technol. 15, 1683-1686 (2004).

[9] K. De Vos, I. Bartolozzi, E. Schacht, P. Bienstman, and R. Baets, "Silicon-on-Insulator microring resonator for sensitive and label-free biosensing," Optics Express, vol. 15, Jun. 2007, pp. 7610-7615. 